Original Research Paper

\title{
Ant Colony Optimization for Load Management Based on Load Shifting in the Textile Industry
}

\author{
${ }^{1}$ Chaimongkon Chokpanyasuwan, ${ }^{2}$ Tika Bunnag and ${ }^{3}$ Ratthasak Prommas \\ ${ }^{1}$ Rattanakosin College for Sustainable Energy and Environment (RCSEE), Rajamangala University of Technology \\ Rattanakosin, 96 moo 3 Puthamonthon Sai 5, Salaya, Puthamonthon, Nakhon Pathom, 73170, Thailand \\ ${ }^{2}$ Pitipak Engineering Co., Ltd. $508 / 12$ Carpark Building Synphaet Hospital Ramindra Rd, \\ Ramindra, Kannayaw 10230, Thailand \\ ${ }^{3}$ Department of Mechanical Engineering, Faculty of Engineering, Rajamangala University of Technology Rattanakosin, \\ (RMUTR), 96 moo 3 Puthamonthon Sai 5, Salaya, Puthamonthon, Nakhon Pathom, 7317, Thailand
}

Article history

Received: 20-11-2014

Revised: 20-12-2014

Accepted: 24-03-2015

Corresponding Author:

Ratthasak Prommas

Department of Mechanical

Engineering, Faculty of

Engineering, Rajamangala

University of Technology

Rattnakosin, (RMUTR), 96

moo 3 Puthamonthon Sai 5,

Salaya, Puthamonthon, Nakhon

Pathom, 73170 Thailand

Email: ratthasak.pro@rmutr.ac.th

\begin{abstract}
The textile industry is a complicated manufacturing industry because it is a fragmented and heterogeneous sector dominated by Small and Medium Enterprises (SMEs). There are various energy-efficiency opportunities that exist in every textile plant. However, even cost-effective options often are not implemented in textile plants mostly because of limited information on how to implement energy-efficiency measures. This paper presents the expansion of problem formulation of consummation management based on load shifting in textile industry. The mathematical model is a Non Polynomial (NP) hard optimization problem to determine the start time of the process in order to minimize the total electricity cost under varying tariffs such as flat rate and Time of Use (TOU). For solve this problem, Ant Colony Optimization (ACO) is applied and compared with Genetic Algorithm (GA) and Particle Swarm Optimization (PSO). To show its efficiency, the case studies in case of Single Process Multiple Jobs (SPMJ) in term of small, medium and large scales are demonstrated. The results show that the proposed method is able to achieve the best solution efficiently and easy to implement.
\end{abstract}

Keywords: Load Management, Time of Use, Ant Colony Algorithm, Textile Industry Optimization

\section{Introduction}

The textile industry is one of the most convoluted contemporary industries because it is a fragmented and heterogeneous sector dominated by Small and Medium Enterprises (SMEs). Characterizing the textile manufacturing industry is composite because of the wide variety of substrates, processes, machinery and apparatus used and finishing steps undertaken. Different types of fibers or yarns, methods of fabric production and finishing processes (preparation, printing, dyeing, chemical/mechanical finishing and coating), all correlate in producing a finished fabric. Electrical energy is one of the main cost factors in the textile industry. Especially in times of high energy price volatility, improving energyefficiency should be a primary concern for textile plants. There are various energy-efficiency opportunities that exist in every textile plant, many of which are costeffective. However, even cost-effective options are not often implemented in textile plants mostly because of limited information on how to implement such energyefficiency measures, especially given the fact that a majority of textile plants are categorized as SMEs and hence they have limited resources to acquire this information. Know-how on energy-efficiency technologies and practices should, therefore, be prepared and disseminated to textile plants. An extensive literature review was conducted in this study to collect information on the energy use in and energy efficiency measures/technologies for the textile industry (Ashok and Banerjee, 2000). In recent years; various Load Management (LM) programs are used by electric utilities to tackle the peak load problem (Ashok and Banerjee, 2003). LM is the strategies, reported with potential reduction in system peak demand with different LM (Sheen et al., 1994). Many the utility, have the reduction in peak demand improves the utilization of base load generating stations and avoids costly peaking stations 
and other device in their service area (Cohen and Wang, 1988). The customer benefits, as there is reduction in the energy cost bill. To promote LM, utilities offer incentives like Time of Use (TOU) rates (Ashok, 2006). The textile plants being the bulk consumers, the contact of LM action like load scheduling under TOU tariff on peak demand reduction is high. The textile industries can also control their energy costs by optimal load schedule under TOU tariff. The production-scheduling problem in textile manufacturing, as a multi-stage job shop scheduling problem, is also reported (Laoboonlur, 2004). Most of the models for textiles are centered on process scheduling to achieve the targeted production subject to production constraints and do not give the impact on peak electricity-demand reduction. The optimal schedule to reduce the peak utility load is reported for production processes considering cost worker and process constraints. Extending this work, a production process model coupled with an optimization formulation for process industries with jobs and continuous type loads is presented here to prescribe optimal load-schedule strategies under different tariff structures. The industries can also control their electricity costs by optimal load schedule under TOU tariff. Load Shifting (LS) is a basic method for adapting load curves so as to reduce marginal costs of electric power supply. Load shifting within textile industry is based on their inhabitant behavior and in their equipment. Thus, we have to account for both (Bremer et al., 2008) the practice of managing electricity supply and demand so that peak energy use is shifted to off-peak periods. Properly done, load shifting helps meet the goals of improving energy efficiency and reducing emissions by smoothing the daily peaks and valleys of energy use and optimizing existing generation assets. Load shifting may be accomplished in several ways. Demand response programmers shift load by controlling the function of machine production, water heaters, heat pumps and similar electric loads at maximum demand times. The goal of this paper is to develop the ACO algorithm for solving the load shifting problem. Firstly, the load scheduling problem consisting of small, medium and large jobs which considers the applied to determine the start time of the process in order to minimize the total electricity cost under varying tariffs such as flat rate and Time of Use (TOU). To show its efficiency, the case studies in case of Single Process Multiple Jobs (SPMJ) are studied. The results optimized by the ACO are compared to those obtained by the conventional approaches, i.e., GA and PSO in terms of solution quality and computational efficiency.

The paper is organized as follows: Section 2 problem formulation deals with the design principles of LS jobs scheduling on machine production. Section 3 describes the basics of Ant Colony System. Section 4 demonstrates the experimental setup deals with the results and discussions and conclusions are drawn in section 5 .

\section{Overview and Problem Formulation}

\section{Textlie Process}

Figure 1 is a generalized flow diagram depicting the various textile processes that are involved in converting raw materials in to a finished product. All of these processes do not occur at a single facility, although there are some vertically integrated plants that have several steps of the process all in one plant. There are also several niche areas and specialized products that have developed in the textile industry which may entail the use of special processing steps that are not shown in Fig. 1:

- Spun yarn spinning

- Weaving

- Wet-processing (preparation, dyeing, printing and finishing)

- Man-made fiber production

\section{Types of Energy Used in Textile Industry}

In general, energy in the textile industry is mostly used in the forms of: Electricity, as a common power source for machinery, cooling and temperature control systems, lighting, office equipment, etc.; oil as a fuel for boilers which generate steam; liquefied petroleum gas; coal; and city gas. The rational use of energy calls for a broad application of energy conservation technologies in the various industrial sectors where energy is wasted. One of these energy intensive industrial sectors to be considered to improve efficiency through the introduction of modern energy conservation technologies is the textile industry. In the textile industry, appreciable amounts of energy could be saved or conserved by regulating the temperature in the steam pipes, adjusting the air/fuel ratio in the boilers and installing heat exchangers using warm waste water.

\section{Load Pattern and Electrical Trariffs in Thailand}

Domestic consumption in 1991 could be divided into three periods of time, i.e., during the evening time hours of $6: 30$ to $9: 30 \mathrm{pm}$ when the power consumption was highest (peak period), during the daytime hours of 8:00 am to $6: 30 \mathrm{pm}$ when the consumption was moderate (partial-peak period) and during the night-time and early morning hours of 9:30 pm to 8:00 am when the consumption was low (off-peak period). During the peak period, the Electricity Generating Authority of Thailand (EGAT) had to use its full generation capacity until the minimum reserve margin was reached; the Provincial Electricity Authority (PEA) had to use its full dispatching capacity in the same period, whereas the Metropolitan Electricity Authority (MEA) had to maximize its dispatching capacity in the afternoon when the consumption nationwide was at moderate level. 


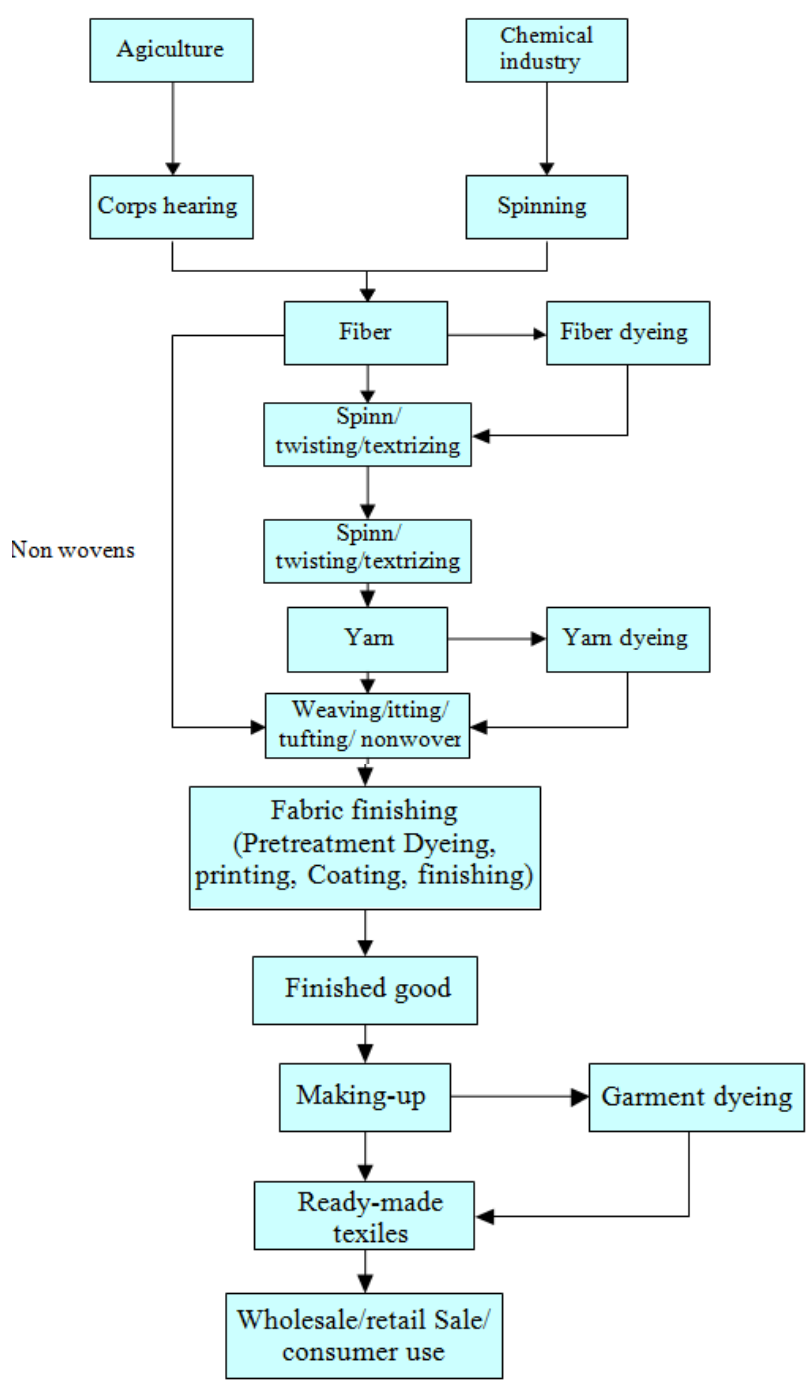

Fig. 1. Textile process

Therefore, if the demand had increased during the peak period, the power sector would have been required to increase the investment in power generation and distribution. Both from an energy efficiency perspective and from equity of rates perspectives, large consumers during the peak period should pay a higher tariff than small consumers during the off-peak period of the system. One way to determine a fair tariff was to use a formula to automatically adjust the electricity charges in different periods of a day, i.e., implement a Time-of-Day (TOD) Rate. However, this method could not be applied to all individual consumers because the expense for meter installation was rather high.

\section{The TOD Rate or Flat Rate}

The TOD Rate is the electricity tariff rate that varies with the time-period during which electricity has been used during the day. Its objective is to shift consumption from the peak to off-peak periods. The TOD Rate was first used in Thailand in 1964 as an alternative rate applied to the industrial sector. In 1966 it became one of the alternative rates for customers that were served directly by EGAT. In practice, only a few customers opted into this rate.

\section{Time-of-Use (TOU) Rate}

The Time-of-Use Rate (TOU) was established as an alternative for users who currently use the TOD rate so that the tariff structure would reflect the costs and load curve of the system. As of September 1998, 167 customers were on TOU rates, of which 11 were in MEA and 156 in PEA. The TOU rate differs from the TOD rate in the following: TOU will increase tariff categories for consumers whose consumption is at or greater than $115 \mathrm{kV}$; the tariff will be lower than that of the previous $69 \mathrm{kV}$. TOU will be divided into two periods of time, i.e., peak, from 9 am to $10 \mathrm{pm}$ on Mondays through Saturdays and off-peak during all other hours. Under the TOU rate, the energy charge varies according to voltage levels and periods of time. Under the TOU rate, there is no demand charge during the Off-Peak period. The newly established TOU rate will be compulsory for new consumers who have to use the TOD rate. The TOU rate best reflects actual costs of the current power system. This will provide more incentives for consumers to change their consumption behavior, whereas those whose consumption is consistent and who do not change their consumption behavior will not be harmed.

The research for electrical tariff to major industrial sector may consist of a charge for the total $\mathrm{kWh}$ consumed, a power factor charge and a $\mathrm{kW}$ maximum demand charge.

The costs of the total electrical energy before shifting and after shifting are calculated with the TOU tariff rate applying by the MEA rated voltage is $24 \mathrm{kV}$ connected grid. The tariff for the industrial sector is TOD rate or late rate as shown Table 1 and TOU rate shown in Table 2.

\section{Load Shifting Problem Formulation}

In this section, the load management based on load shifting for the textile process is presented. The problem can explain in 3 parts; systematic model, feasible solution structure, fitness function.

\section{Systematic Model}

Systematic model of load shifting for the textile process can be explained in four parts which are input parameters, decision variables, constraints and system output as shown in Fig. 2.

\section{Input Parameters}

The input parameters consist of the step of textile production process, process and transfer time, number of machine and man power of each step, rated power each of machines. The input varies depending on the of production process type. 


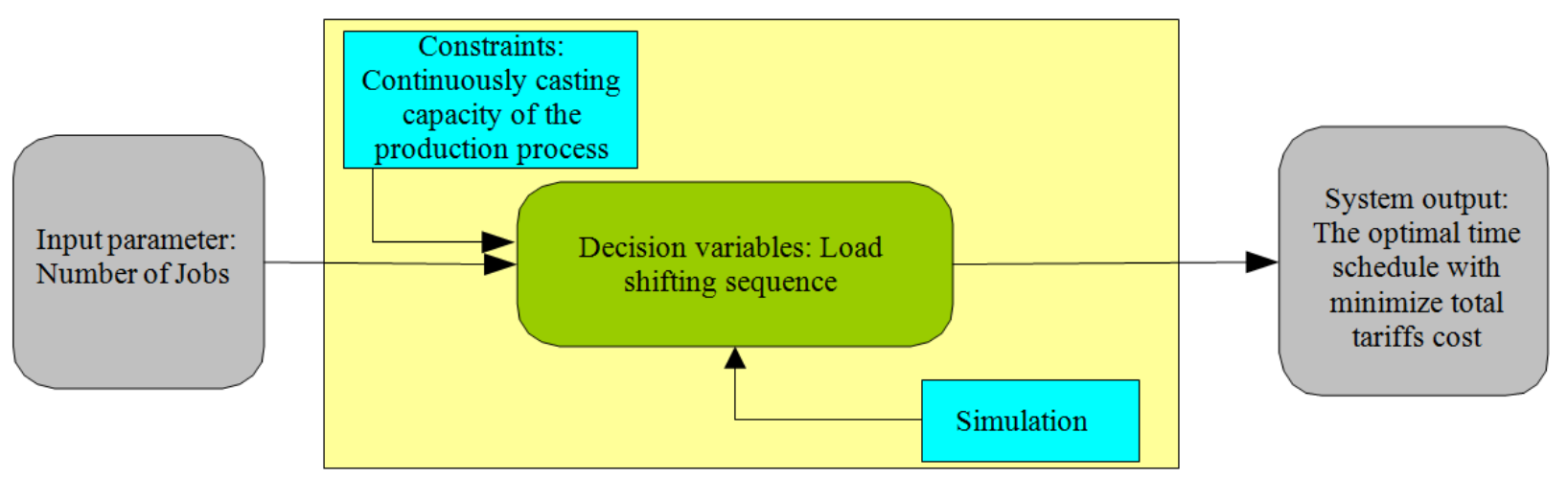

Fig. 2. Systematic model of load shifting problem

Table 1. The Time-of-Day (TOD) rate in Thailand

\begin{tabular}{lllll}
\hline Time of day & Demand charge $(\mathrm{Baht} / \mathrm{kW})$ & $3 *$ & $\begin{array}{l}\text { Energy charge } \\
(\mathrm{Baht} / \mathrm{kWh})\end{array}$ \\
rate (Tariff) & $-1 *$ & $2 *$ & 0 & 1.6660 \\
\hline $69 \mathrm{kV}$.and over & 224.30 & 29.91 & 0 & 1.7034 \\
$12-24 \mathrm{kV}$. & 285.05 & 58.88 & 0 & 1.7314 \\
Below $12 \mathrm{kV}$ & 332.71 & 68.22 & 0 & 0 \\
\hline
\end{tabular}

Flat rates or Time-of-Day (TOD Tariff): Demand charge: 1.7034 Bath $/ \mathrm{kWh}$ or $(0.052 \$ / \mathrm{kWh})$ ), Energy charge: $58.88 \mathrm{Bath} / \mathrm{kW}$ or $(1.84 \$ / \mathrm{kWh})$ ), (Note: Present transaction rate 1 US\$:bath. 32.0

*On Peak: Everyday from 06.30 to 09.30 PM,* Partial Peak: Everyday from 08.00 to 06.30 PM (only the amount of maximum demand that is out of the On-Peak period will be charged at this rate).

*Off Peak: Everyday from 09.30 to 08.00 AM (No demand charge)

Table 2. Time of use tariff (TOU tariff)

\begin{tabular}{|c|c|c|c|c|}
\hline \multirow[b]{2}{*}{$\begin{array}{l}\text { Time of } \\
\text { use (Tariff) }\end{array}$} & \multicolumn{3}{|c|}{ Demand charge $($ Baht $/ \mathrm{kW})$ Energy charge $(\mathrm{Baht} / \mathrm{kWh})$} & \multirow[b]{2}{*}{$\begin{array}{l}\text { Service charge } \\
\text { (Baht/month) }\end{array}$} \\
\hline & $1 *$ & $1^{*}$ & $2 *$ & \\
\hline $69 \mathrm{kV}$ and over & 74.14 & 2.6136 & 1.1726 & 228.17 \\
\hline $12-24 \mathrm{kV}$ & 132.93 & 2.6950 & 1.1914 & 228.17 \\
\hline Below $12 \mathrm{kV}$. & 210.00 & 2.8408 & 1.2246 & 228.17 \\
\hline
\end{tabular}

Time of Use tariff (TOU tariff), Demand Charge: 132.93 Bath $/ \mathrm{kW}$ or $(4.15 \$ \mathrm{~kW})$, Energy Charge: 1.1914 Bath $/ \mathrm{kWh}$ or $(0.037 \$ / \mathrm{kWh})$, On Peak: Monday-Friday from 09.00 to 10.00 PM off Peak: Monday-Friday from 10.00 to 09.00 AM, SaturdaySunday and normal public holiday (excluding substitution holiday) from 00.00 AM to 12.00 PM.

*On Peak: Monday-Friday from 09.00 to 10.00 PM,*Off Peak: Monday-Friday from $10.00 \quad$ PM to $09.00 \quad$ AM Saturday-Sunday and normal public holiday (excluding substitution holiday) from 00.00 to $12.00 \mathrm{PM}$.

\section{Decision Variables}

The starting time of each job are assigned to decision variable. This variable will be used to determine the time schedule to minimize total energy cost.

\section{Production Constraints}

Normally, the textile industries have the specific machine and man power. The process cannot operate if the machine and man power are not available. Therefore, the constrains of time dispatching are limited by the of machine and manpower of industry.

\section{System Output}

The objective of load shifting scheduling is to minimize the total energy cost of process time schedule.

\section{Structure and Feasible Solution}

In this study, the structure of an solution for load shifting problem is composed of a set of starting time number each job. Therefore, size of solution is $1 \times n$ matrix where $\mathrm{n}$ is number of jobs. Note that it is very important to create a set of solution satisfying the constraints it is called a feasible solution.

The feasible solution of candidate solution is related to the of possible jobs, which can be found by

$$
F S=T^{n}
$$

Where:

FS $=$ Number of feasible solution

$T \quad=\quad$ Number of time periods. 


\section{Fitness Function}

In order to evaluate fitness function of load shifting problem, firstly define indices, parameters and decision variables as follows.

Indices:

$i$ : Index of job, $j \in\{1, n\}$

$j$ : Index of the production process, $j \in 1, m\}$

$t$ : Index of load shifting time schedule from job $i$ to production process $j, t \in 1, n\}$

Variables:

$M: \quad$ Number machine of production process.

$n: \quad$ Number of job.

$P T_{j}: \quad$ Process time of production process $j$

$N M_{j}$ : $\quad$ Number of machines of production process $j$

$N E_{j}: \quad \quad \quad$ Number of manpower of production process $j$

$T T_{j}: \quad$ Transfer time of production process $j$

$M P_{j}: \quad$ Rated power machine of production process $j$

$S T_{j}: \quad \quad$ Start time of job $i$

$S T j_{j-1}: \quad$ Stop time of job ${ }_{j-1}$

Start_time $_{i j}$ : Start time of job $i$ and process $j$

Stop_time $i j$ : Stop time of job $i$ and process $j$

$T: \quad$ Time interval

$R_{T}$ : $\quad$ Tariffs cost rate of time interval

The Start time $_{i j}$ is the present time Start time $e_{i j}$ is the time at which the $m_{n}$ machines are set operational:

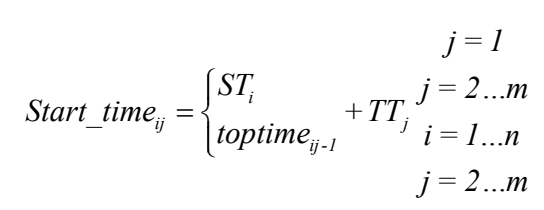

The Start_time $e_{i j}$ is the stop present time; Stop_time $i j$ is the time at which the $m_{n}$ machines are not set operational as:

$$
\text { Stop }_{\text {time }_{e_{j}}}=\text { Start }_{\text {time }_{i j}}+P T_{j}
$$

Total energy consumption of machines is obtained by summing the energy consumptions of all machines operating in the textile manufacturer. Certain machines processes have the feature of having multiple duty cycles. These machines have the capability to operate for different levels of power consumption. Energy consumption of given equipment for a particular period of time can be stated as:

$$
E_{N M j}=P_{N M j} \times T \times R_{T}
$$

where, $E_{N M j}$ is the energy consumption of the $N M_{i j}$ machines $P_{N M J}$ is power consumption at $T$ time interval is the time at which the $N M_{j}$ machines are set operational. The cost of electricity consumption of given equipment is dependent on various factors including locality, watts, time of consumption and the cost-rate (peak versus non-peak hour) for that particular time of the day. The procedure of fitness function is given as follow. The information data for load shifting management problem is shown in Table 3 . Here, the production process has 10 steps for plant textile in Thailand. This information will be used to convert a feasible solution or sequence to a time schedule for reduces energy power consumption.

Step1: Determine starting time and stop time of job $i$ and process $j$. The step to find starting time of job $i$ start with the computing Equation (2) and to find stop time as Equation (3)

Step2: Determine time interval of each process and evaluate rate of tariff based on Tariff of use.

Step3: Evaluate tariff cost by Equation (4) and energy cost of process at time interval

\section{Case Study}

To illustrate the model proposed, a case study has been worked out for a typical the dyeing machines of production process for 10 steps of textile process Samutsakorn province in Thailand. The connected load of the plant is $22 \mathrm{kV}$ and transformer rated power is $2500 \mathrm{kVA}, 0.400 / 230 \mathrm{~V}$. Average daily electricity consumption is $2200 \mathrm{kWh}$. The plant has an automatic power factor correction unit which maintains the power factor nearly unity. For Example, assumed a solution is $[8,8,8]$, First step, form Table 3, the column number of machines of plant defined as [2, 3,4,2,4,2,2,4,3,4] transform given by matrix $4 * 10=$ $\left[\begin{array}{llllllllll}1 & 1 & 1 & 1 & 1 & 1 & 1 & 1 & 1 & 1 \\ 1 & 1 & 1 & 1 & 1 & 1 & 1 & 1 & 1 & 1 \\ 0 & 1 & 1 & 0 & 1 & 0 & 0 & 1 & 1 & 1 \\ 0 & 0 & 1 & 0 & 1 & 0 & 0 & 1 & 0 & 1\end{array}\right]$.

Checked condition, If the total status of machine job $i$ and process the column shown status is $1 \geq 1$, status is available, but the total status of machine job $i$ and process is $=0$, defined the machine cannot operated.

Step 2, determine starting time and stop time of job $i$ and process $j$. The step to find starting time of job $i$ start with the computing Equation 5:

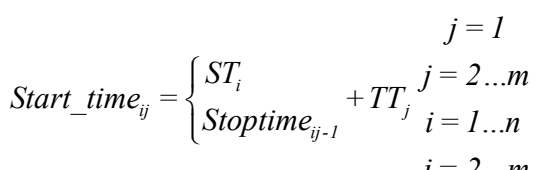


Table 3. The dyeing machines of production process for 10 steps

\begin{tabular}{llllr}
\hline Steps & Process time $(\mathrm{min})$ & No. Machine plant & Transfer time & Machine kW/No. \\
\hline Fabrics prepare & 75 & 2 & 15 & 84 \\
Dyeing, thies & 60 & 3 & 10 & 105 \\
Preset & 100 & 4 & 15 & 123 \\
Fabric prepare & 90 & 2 & 10 & 94 \\
Dying & 150 & 4 & 20 & 117 \\
Ring spinning & 130 & 2 & 10 & 100 \\
Rewinding & 60 & 2 & 15 & 94 \\
Heat setting & 100 & 4 & 20 & 134 \\
Inspection & 60 & 3 & 10 & 78 \\
Packing & 95 & 4 & 15 & 85
\end{tabular}

(Table 3: Shown the textile process in Thailand)

Table 4. Time Scheduled of jobs 1, 2, 3 for load shifting technique

\begin{tabular}{lllllllllll}
\hline Jobs process & 1 & 2 & 3 & 4 & 5 & 6 & 7 & 8 & 9 & 10 \\
\hline Job \# 1 & & & & & & & & & & \\
Start time & $8: 00$ & $9: 30$ & $10: 40$ & $12: 35$ & $14: 15$ & $17: 05$ & $19: 25$ & $20: 40$ & $22: 40$ & $23: 50$ \\
Stop time & $9: 15$ & $10: 30$ & $12: 20$ & $14: 05$ & $16: 45$ & $19: 15$ & $20: 25$ & $22: 20$ & $23: 40$ & $01: 25$ \\
Job \# 2 & & & & & & & & & \\
Start time & $8: 00$ & $9: 30$ & $10: 40$ & $12: 35$ & $14: 15$ & $17: 05$ & $19: 25$ & $20: 40$ & $22: 40$ & $23: 50$ \\
Stop time & $9: 15$ & $10: 30$ & $12: 20$ & $14: 05$ & $16: 45$ & $19: 15$ & $20: 25$ & $22: 20$ & $23: 40$ & $01: 25$ \\
Job \# 3 & & & & & & & & & & \\
Start time & $9: 30$ & $11: 00$ & $12: 10$ & $14: 15$ & $15: 55$ & $19: 25$ & $21: 45$ & $23: 00$ & $01: 00$ & $02: 10$ \\
Stop time & $10: 45$ & $12: 00$ & $13: 50$ & $15: 45$ & $18: 25$ & $21: 35$ & $22: 45$ & $00: 40$ & $02: 00$ & $03: 45$ \\
\hline
\end{tabular}

Table 5. The result of energy cost power consumptions load-shifting technique for case study

\begin{tabular}{|c|c|c|c|c|c|c|c|c|c|c|c|}
\hline Job process & 1 & 2 & 3 & 4 & 5 & 6 & 7 & 8 & 9 & 10 & $\begin{array}{l}\text { Total energy } \\
\text { cost (Baht) }\end{array}$ \\
\hline Job1 & 155.6762 & 282.9750 & 552.4750 & 379.9950 & 788.2875 & 583.9167 & 253.3300 & 534.7225 & 92.9292 & 160.3426 & 3785.6 \\
\hline Job2 & 155.6762 & 282.9750 & 552.4750 & 379.9950 & 788.2875 & 583.9167 & 253.3300 & 534.7225 & 92.9292 & 160.3426 & 3785.6 \\
\hline Job3 & 282.9750 & 282.9750 & 552.4750 & 379.9950 & 788.2875 & 583.9167 & 147.3262 & 266.0793 & 92.9292 & 160.3426 & 3537.3 \\
\hline $\begin{array}{l}\text { Total energy } \\
\text { cost (Baht) }\end{array}$ & & & & & & & & & & & 11109.5 \\
\hline
\end{tabular}

If process can not available to find stop time as equation, minimize:

$$
\left(\text { Stop_time } e_{k i}\right) i=1 \ldots . n, k^{1} i
$$

The results of this step are shown in Table 4.

Step3: Evaluate tariff cost by Equation (4) and energy cost of process at interval time. The results of this step are shown in Table 5.

\section{Ant Colony Optimization}

Inspired by the collective behavior of a real ant colony, Marco Dorigo first introduced the Ant System (AS) in his Ph.D. thesis (1992) and Dorigo et al. (1996) further continued the study (Colorni et al., 1991). The characteristics of an artificial ant colony include positive feedback, distributed computation and the use of a constructive greedy heuristic. Positive feedback accounts for rapid discovery of good solutions, distributed computation avoids premature convergence and the greedy heuristic helps to find acceptable solutions in the early stages of the search process. In order to demonstrate the AS approach, the authors apply this approach to the classical TSP, asymmetric TSP, Quadratic Assignment Problem (QAP) and job-shop scheduling. The AS shows very good results in each applied area. More recently, Dorigo and Gambardella have worked on extended versions of the AS paradigm. ACO is the one of the extensions and has been applied to the symmetric and asymmetric TSP with excellent results (Dorigo et al., 1996). The Ant System has also been applied with success to other combinatorial optimization problems such as the vehicle routing problem (Dorigo and Gambardella, 1997a; 1997b).

ACO is an algorithm that was inspired by the behavior of real ants. Ethnologists have studied how blind animals such as ants capable of finding the shortest path from food sources to the nest without using visual cues. They are also capable of adapting to changes in the environment. For example, finding a new shortest path once the old one is no longer feasible due to a new obstacle. The studies of ethnologists reveal that such capabilities are essentially due to communicating information among individuals regarding path to decide 
the direction. Ants deposit a certain amount of pheromone while walking and each ant probabilistically prefers to follow a direction rich in pheromone rather than a poorer one.

The process can be clearly illustrated by Fig. 3a ants are on a straight line that connects a food source to their nest. An ant will deposit pheromone while walking and it probabilistically prefers to follow a direction rich in pheromone.

This behavior can be explained how ants can find the shortest path that reconnects a line that is broken by an obstacle in Fig. 3b. On introducing, those ants are just in front of the obstacle and they cannot continue to go. Therefore, they have to choose between turning right or left. Half the ants choose to turn right and the other half choose to turn left. A similar situation arises on the other side of the obstacle Fig. 3c. Ants choose the shorter path will more rapidly reconstitute the interrupted pheromone trail compared with those choosing the longer path. Thus, the shorter path will receive a greater amount of pheromone per time unit and, in turn, lager number of ants will choose the shorter path. Due to this positive feedback, all the ants will rapidly choose the shorter path Fig. 3d. All ants move approximately the same speed and deposit a pheromone trail at approximately the same rate. The time consumed on the longer side of an obstacle is greater than the shorter path. It thus makes the pheromone trail accumulate more quickly on the shorter path. Moreover, ants prefer higher number of pheromone trail that causes accumulation can be built up faster on the shorter path.

In general, the procedure of ACO algorithm can be described as follows: $m$ ants are initially positioned at the nest. Each ant will choose a possible route as a solution. In fact, each ant builds a feasible solution (called a tour) by a stochastic greedy search, called; the state transition rule is repeatedly applied. Once all ants have terminated their tours, the following steps are performed.

The amount of pheromone is modified by applying the global updating rule. Ants are guided, in building their tours, by both heuristic information and pheromone information. Naturally, a link with a high amount of pheromone is a desirable choice. The pheromone updating rules are designed so that they tend to give more pheromone to edges, which should be visited by ants.

A flowchart of a conventional ACO algorithm is shown in Fig. 4.

The detail of ACO algorithm can be described in the following steps:

Step 1 Initialization

Set $\mathrm{NC}=0 \quad / * \mathrm{NC}$ : cycle counter */

For every combination $(\mathrm{i}, \mathrm{j})$

Set an initial value $\tau_{i j}(0)=\tau_{0}$ and $\Delta \tau_{i j}=0$

End
Step 2 Construct feasible solutions

For $\mathrm{k}=1$ to $\mathrm{m} / * \mathrm{~m}$ : Number of ants */

For $\mathrm{i}=1$ to $\mathrm{n} / * \mathrm{n}$ : Number of links */

Choose a level of connection with transition probability given by Equation (6).

End

Calculate fitness function $f_{k} / * \mathrm{f}_{\mathrm{k}}$ : Fitness value of each ant $* /$

End

Update the best solution

Step 3 Global updating rule

For every combination $(\mathrm{i}, \mathrm{j})$

For $\mathrm{k}=1$ to $\mathrm{m}$

Find $\Delta \tau_{i j}^{k}$ according to Equation (10)

End

Update $\Delta \tau_{i j}$ according to Equation (9)

End

Update the trail values according to Equation (9)

Update the transition probability according to Equation (6)

Step 4 Next search

Set $\mathrm{NC}=\mathrm{NC}+1$

For every combination $(\mathrm{i}, \mathrm{j})$

$\Delta \tau_{i j}=0$

End

Step 5 Termination

If $\left(\mathrm{NC}<\mathrm{NC}_{\max }\right)$

Then

Goto step 2

Else

Print the best feasible solution

Stop

End

End

\section{State Transition Rule}

The state transition rule of the ant colony is given in Equation (6). This equation represents the probability that ant $k$ selects a link connects node $i$ and node $j$ :

$$
p_{i j}^{k}(t)=\frac{\left[\tau_{i j}(t)\right]^{\alpha}\left[\eta_{i j}(t)\right]^{\beta}}{\sum_{m=1}^{M_{i}}\left[\tau_{i m}(t)\right]^{\alpha}\left[\eta_{i m}(t)\right]^{\beta}}
$$

where, $\tau_{i j}$ is the pheromone intensity and $\eta_{i j}$ is the heuristic information between node $i$ and node $j$, respectively. In addition, $\alpha$ is the relative importance of the trail and $\beta$ is the relative importance of the heuristic information. The problem specific heuristic information is:

$\eta_{i j}=\frac{1}{C_{i j}}$

where, $C_{i j}$ represents the associated cost. Therefore, the level of link with smaller cost has greater probability to be chosen. 


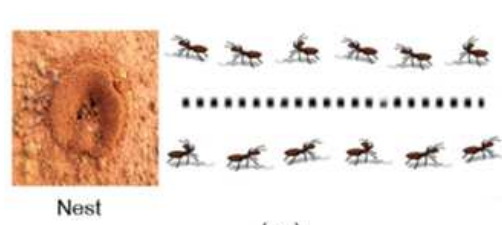

(a )

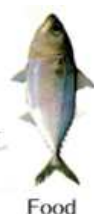

Food

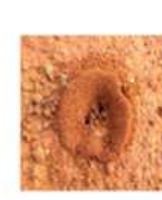

Nest

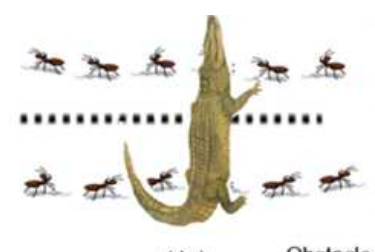

(b) Obstacle

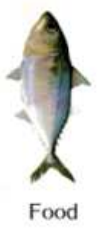

Food

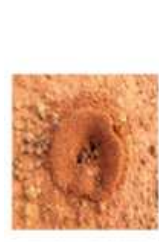

Nest

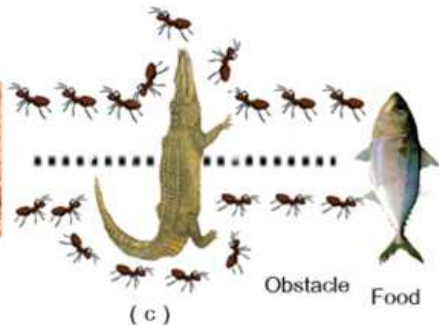

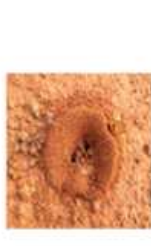

Nest

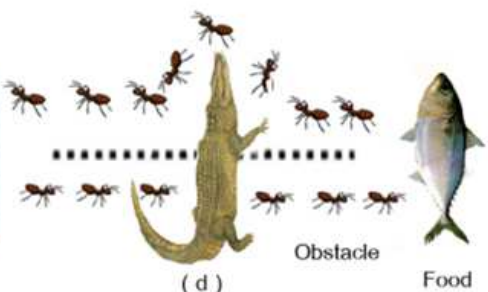

Fig. 3. Behavior of ants; (a) Real ants follows a path between nest and food source. (b) An obstacle appears on the path: Ants choose whether to turn left or right with equal probability. (c) Pheromone is deposited more quickly on the shorter path. (d) All ants have chosen the shorter path

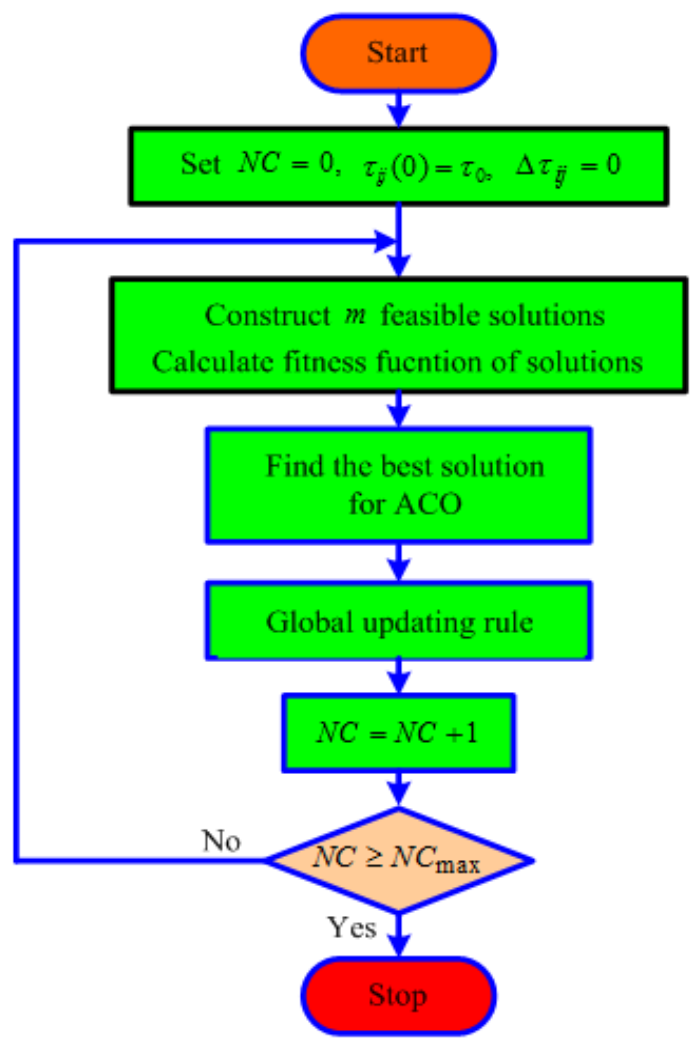

Fig. 4: Flow chart of conventional ACO algorithm

\section{Global Updating Rule}

During the solution construction, it is no guarantee that an ant will construct a feasible solution, which obeys the reliability constraint. The pheromone updating treats the unfeasible solution. The amount of pheromone, deposited by ants, is set to a high value if the generated solution is feasible. On other hand, this value is set to a low value if it is infeasible. Therefore, this value depends on the solution quality. Infeasibility can be handled by assigning the penalty which proportion to the amount of reliability violations. In case of feasible solution, an additional penalty is introduced to improve its quality.

Following the above remarks, the trail intensity is updated as follows:

$\tau_{i j}(n e w)=\rho \tau_{i j}($ old $)+\Delta \tau_{i j}$

$\rho$ is a coefficient such that $(1-\rho)$ represents the evaporation of trail and $\Delta \tau_{i j}$ is:

$\Delta \tau_{i j}=\sum_{k=1}^{m} \Delta \tau_{i j}^{k}$

where, $m$ is the number of ants and $\Delta \tau_{i j}^{k}$ is given by:

$$
\begin{aligned}
& \Delta \tau_{i j}^{k} \\
& =\left\{\begin{array}{cc}
Q \cdot \text { penalty }_{k} \cdot C_{k} & \text { if the } \mathrm{k}^{\text {th }} \text { ant chooses level } j \text { for link } i \\
0 & \text { otherwise }
\end{array}\right.
\end{aligned}
$$

where, $Q$ is a positive number and penalt $\mathrm{y}_{\mathrm{k}}$ is defined as follows:

$$
\text { penalty }_{k}=\left(\frac{C_{k}}{C^{*}}\right)^{a}
$$

$C_{k}$ is the cost obtained by the $\mathrm{k}^{\text {th }}$ ant, $\mathrm{C}^{*}$ is the best obtained solution. Parameter a represents the relative importance of penalties. 


\section{Experiment Results and Discussions}

To assess the feasibility solution of the swarm intelligence techniques i.e., GA, PSO and ACO, three take load sizes problems (small, medium and large problems) are applied. All optimization methods (GA, PSO and ACO) were implemented in MATLAB ${ }^{\circledR}$ package and the simulation cases done on a Intel Core i5-430M, 2.26GHz laptop computer with 4 GB RAM under Windows 8 .

Each studied systems was run 30 times with differential random initial solutions. In order to evaluate the performance of each technique, the best, worst, average and standard deviation of the generation costs and the average of computational time to get near optimum solution are used for evaluation.

Unfortunately, the number of initial solutions and the computational time for an iteration of these techniques are different. Therefore, the execution of search was done by the same iterations. This method is not suitable for comparison. Consequently; this work uses the time limit as the stopping criterion. The search processes of all techniques are executed by the same time period; therefore, the maximum iteration of each technique depends on the time limit. When the search satisfies the stopping criterion, the best, worst, average and standard deviation of the generation costs and the average of computational time to get near optimum solution are reported.

\section{Case Studies}

There are 3, 7 and 10 jobs of dyeing production process of a textile industry. These processes consist of 10 steps as shown in Table 1. That needs to find the starting time of each job. So, the number of the feasible solutions is $1.3824 \times 10^{4}, 4.5865 \times 10^{9}$ and $6.3403 \times 10^{13}$ respectively.

\section{Simulation Results}

After performed 30 trials, the best solutions of three methods are given in Table 6-8. The results of the ACO method in comparison with those of GA and PSO in terms of maximum, average, minimum generation cost, the standard deviation and average computational time are provided. Clearly, the ACO method has always better solutions than other methods.

\section{Comparison of Three Methods}

\section{Solution Quality}

For three size production cost 3,7 and 10 jobs production process for $10 \mathrm{steps}$, the best solutions of three methods are given in Table 6-8 after performed 30 trials. The results of the ACO method are compared with those obtained by GA and PSO in terms of maximum, average, minimum tariffs cost, the standard deviation and average computational time, as provided. Obviously, all methods have succeeded in finding the near optimum solution presented in with a high probability. In order to demonstrate the efficiency of the ACO method, the distribution outlines of the best solution of each trial are considered. Figure 5-7 shows the distribution outlines of the best solution of each trial in case of 3,7 and 10 jobs for production process 10 steps. The Table 9 are result after load shifting 3,7,10 jobs comparative flat rate for 3, jobs more than 3 jobs TOU tariffs cost $29.306 \%$; 7 jobs saving is $25.120 \%$ and 10 jobs to be saving $21.57 \%$. Figure 7,8 and 10 shows the distribution outlines of the best solution of each trial in case of 3,7,10 jobs for production process 10 steps. Almost all tariffs costs obtained by the ACO method are lower. This verifies that the ACO method has better quality of solution. Almost all tariffs costs obtained by the ACO method are lower. This verifies that the ACO method has better quality of solution.

Table 6. Best results obtained 10 steps for small jobs (3 jobs) production process system using GA, PSO, and ACO

\begin{tabular}{lllllll}
\hline Methods & $\begin{array}{l}\text { Max cost } \\
\text { (Baht) }\end{array}$ & $\begin{array}{l}\text { Average cost } \\
\text { (Baht) }\end{array}$ & $\begin{array}{l}\text { Min cost } \\
\text { (Baht)a }\end{array}$ & SD & $\begin{array}{l}\text { CPU time } \\
\text { (sec) }\end{array}$ & $\begin{array}{l}\text { Get } \\
\text { optimum }\end{array}$ \\
\hline PSO & $7,836.41$ & $7,836.41$ & $7,836.41$ & 0.0000000000027751 & 1.892 & 100 \\
GA & $7,836.41$ & $7,836.41$ & $7,836.41$ & 0.0000000000027751 & 1.382 & 100 \\
ACO & $7,836.41$ & $7,836.41$ & $7,836.41$ & 0.0000000000027751 & 1.069 & 100 \\
\hline
\end{tabular}

Table 7. Best results obtained 10 steps for medium jobs ( 7 jobs) production process system using GA, PSO, and ACO

\begin{tabular}{lllllll}
\hline Methods & $\begin{array}{l}\text { Max cost } \\
\text { (Baht) }\end{array}$ & $\begin{array}{l}\text { Average cost } \\
\text { (Baht) }\end{array}$ & $\begin{array}{l}\text { Min cost } \\
\text { (Baht) }\end{array}$ & SD & $\begin{array}{l}\text { CPU time } \\
(\mathrm{sec})\end{array}$ & $\begin{array}{l}\text { Get } \\
\text { optimum }\end{array}$ \\
\hline PSO & $18,950.20$ & $18,321.20$ & $18,066.20$ & 272.30 & 96.666 & 33.33 \\
GA & $18,192.50$ & $18,078.70$ & $18,066.20$ & 0.00213168 & 26.922 & 90.00 \\
ACO & 18,310 & $18,074.30$ & $18,066.20$ & 44.515 & 19.904 & 96.66 \\
\hline
\end{tabular}

Table 8 . Best results obtained 10 steps for large jobs(10 jobs) production process system using GA, PSO, and ACO

\begin{tabular}{lllllll}
\hline Methods & $\begin{array}{l}\text { Max cost } \\
\text { (Baht) }\end{array}$ & $\begin{array}{l}\text { Average cost } \\
\text { (Baht) }\end{array}$ & $\begin{array}{l}\text { Min cost } \\
(\text { Baht })\end{array}$ & SD & $\begin{array}{l}\text { CPU time } \\
(\mathrm{sec})\end{array}$ & $\begin{array}{l}\text { Get } \\
\text { optimum }\end{array}$ \\
\hline PSO & $27,962.90$ & $27,027.30$ & 26379.00 & 568.84 & 121.34 & 0 \\
GA & $26,717.00$ & $26,268.00$ & $26,054.00$ & 0.00856645 & 302 & 0 \\
ACO & $26,232.40$ & $26,035.10$ & $25,912.50$ & 0.00296387 & 0.83 & 16.666 \\
\hline
\end{tabular}


Table 9. The Result for 3, 7 and 10 jobs of load-shifting technique for case study

\begin{tabular}{llll}
\hline Job process & Flat rate cost (Baht) & After shifting with TOU tariffs cost (Baht) & $\%$ Saving \\
\hline 3jobs & 11,085 & $7,836.41$ & 29.306 \\
7 jobs & 24,127 & $18,066.20$ & 25.120 \\
10jobs & 33,040 & $25,912.50$ & 21.570 \\
\hline
\end{tabular}

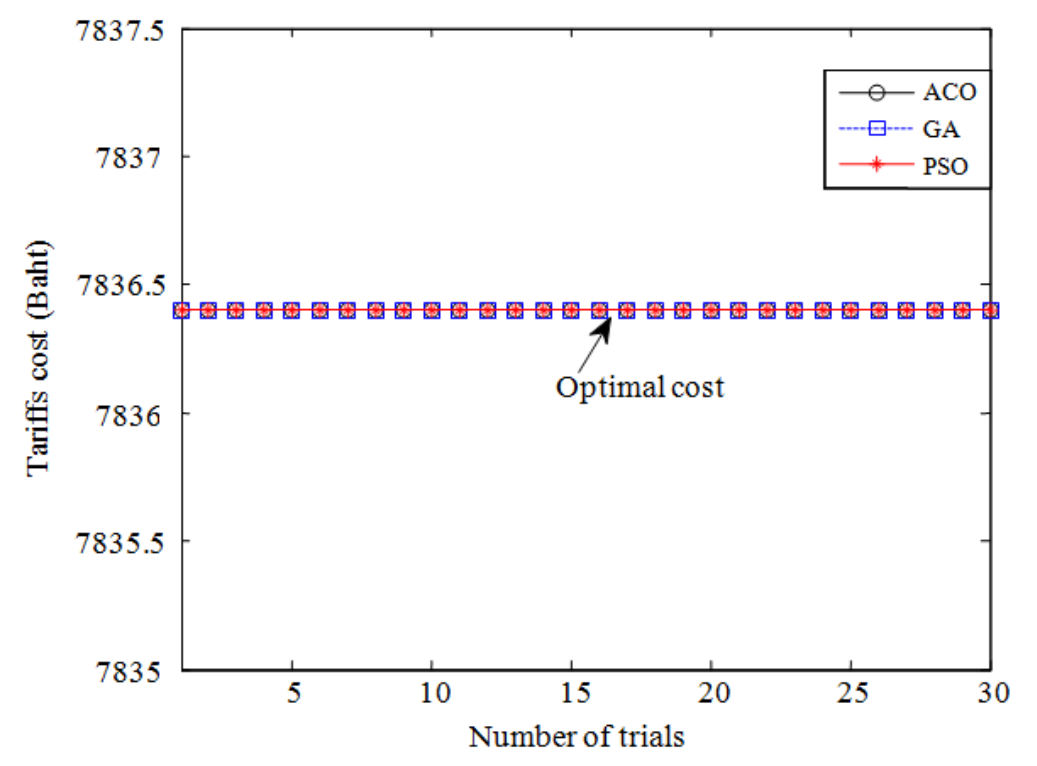

Fig. 5. Distribution of tariffs cost of three methods of 3 jobs for 10 step production process system

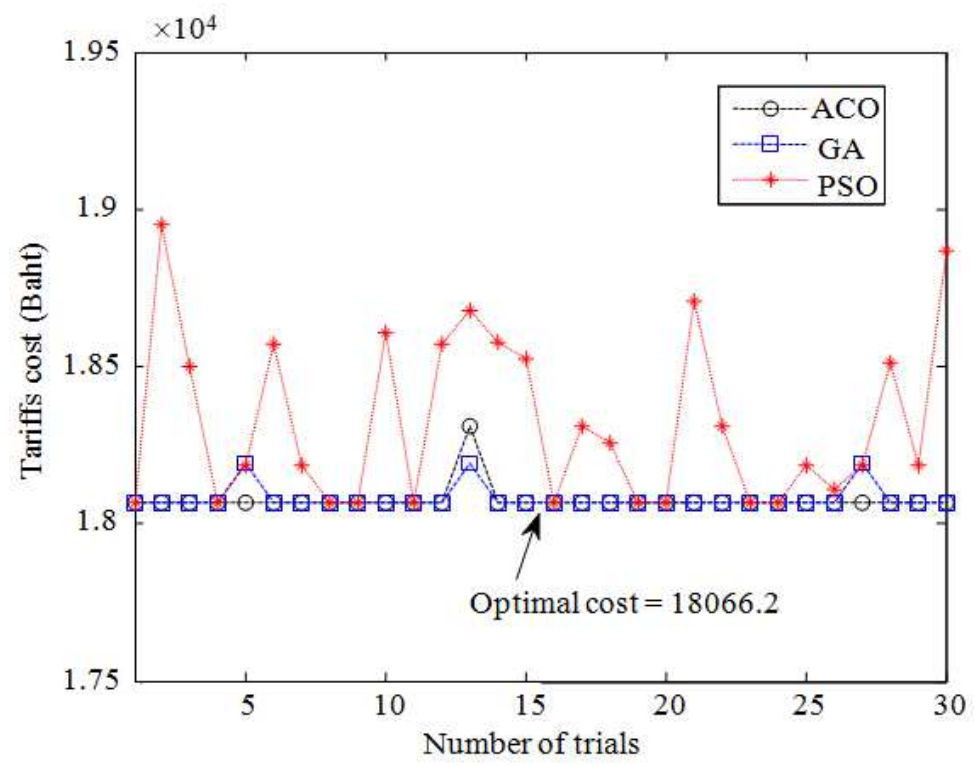

Fig. 6. Distribution of tariffs cost of three methods of 7 jobs of 10 steps production process system

\section{Computation Efficiency}

The convergent characteristics of the ACO compared with other methods for both case studies are shown in Fig. 8-10 Clearly, the ACO converges to the optimum solution much faster than other methods. Furthermore, the comparisons of computation time of five methods for three studies system are already given in Table 6, 7 and 10. The ACO method has better quality of solution. 


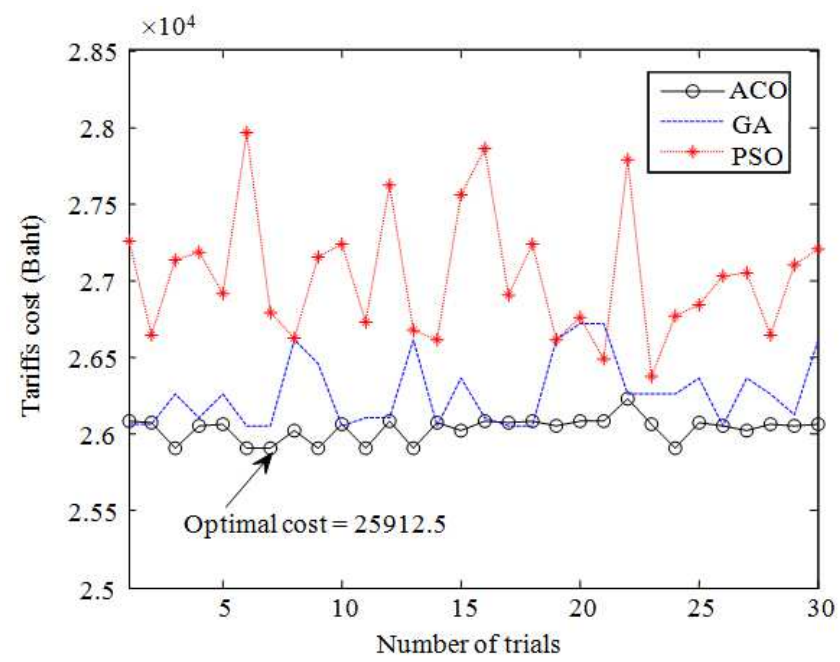

Fig. 7. Distribution of tariffs cost of three methods of 10 jobs for 10 step production process system

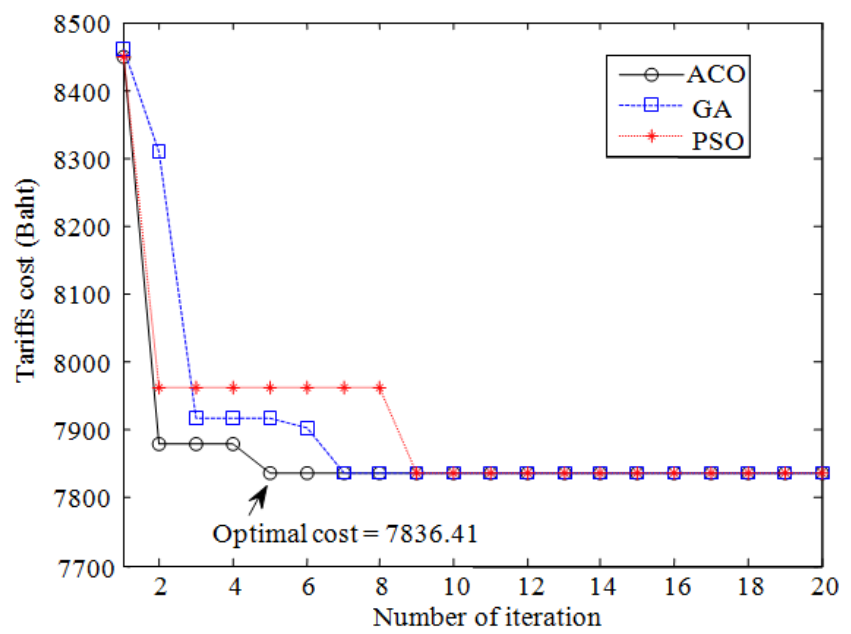

Fig. 8. Convergence curve three methods of 3 jobs for 10 steps production process system

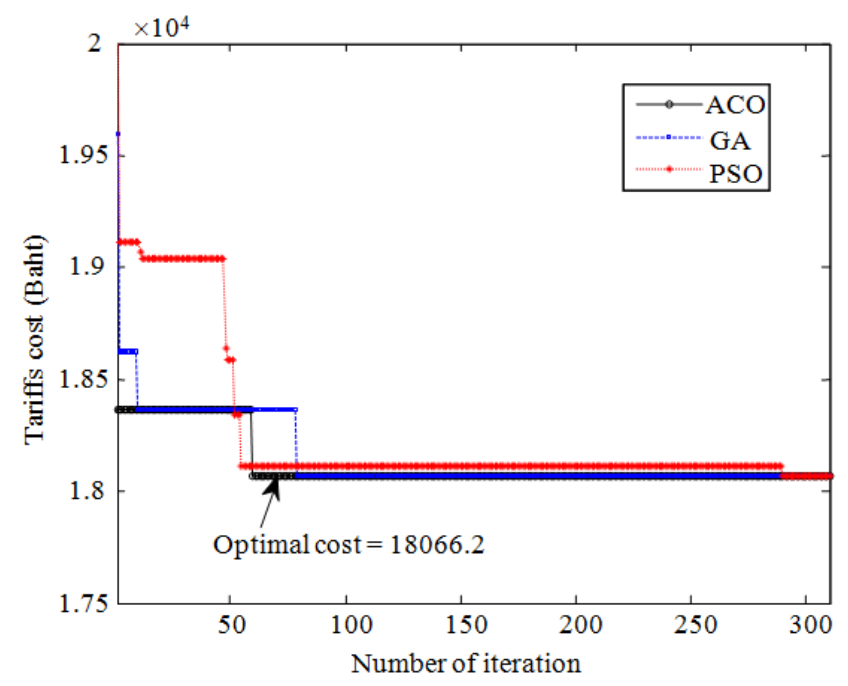

Fig. 9. Convergence curve three methods of 7 jobs for 10 steps production process system 


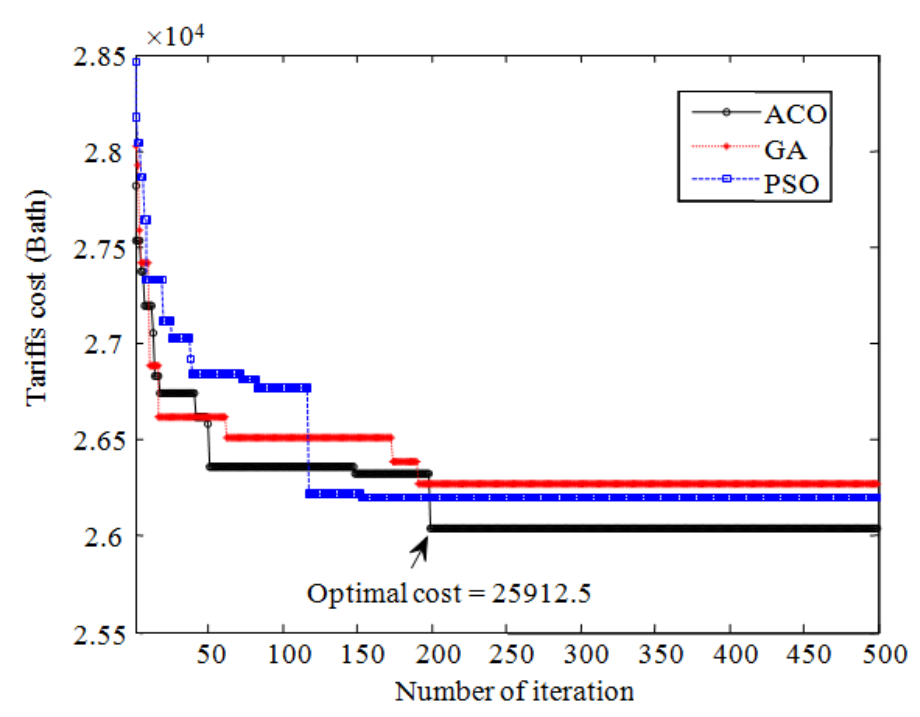

Fig. 10. Convergence curve three methods of 10 jobs for 10 steps production process system

\section{Conclusion}

In this study, the ACO algorithm has been proposed to solve the LS problem by taking the three size problem (small, medium and large scale) and load shifting effects into the case studies in case of Single Process Multiple Jobs (SPMJ) to minimize the total electricity cost under varying tariffs such as flat rate and Time of Use (TOU) consideration. Numerical testing and a comparative analysis show that the proposed ACO algorithm outperforms other methods i.e., GA and PSO in terms of high-quality solution, stable convergence characteristic and good computation efficiency.

\section{Acknowledgement}

The gratefully acknowledge the provision of Green Energy Department of Y.R.C. Textile Co., Ltd in Thailand and Rattanakosin College for Sustainable Energy and Environment (RCSEE), Rajamangala University of Technology Rattanakosin, researchers gratefully acknowledge the provision of Scientific and Technological Research Equipment.

\section{Funding Information}

This study was done by self-financing.

\section{Author's Contributions}

Authors equally contributed to this article.

\section{Ethics}

This article is original research and contains unpublished material. The corresponding author confirms that all of the other authors have read and approved the manuscript and no principled issues concerned.

\section{References}

Ashok, S. and R. Banerjee, 2000. Load-management applications for the industrial sector. Applied Energy, 66: 105-111.

Ashok, S. and R. Banerjee, 2003. Optimal cool storage capacity for load management. Energy, 28: 115-126.

Ashok, S., 2006. Peak-load management in steel plants. Applied Energy, 83: 413-424.

Bremer, J., S. Andreßen, B. Rapp, M. Sonnenschein and M. Stadler, 2008. A modelling tool for interaction and correlation in demand-side market behavior. Proceedings of the 1st European on Energy Modelling using Agent Based Computational Economic, (BCE' 08), pp: 77-91.

Cohen, A.I. and C.C. Wang, 1988. An optimization method for load management scheduling. IEEE Trans. Power Syst., 3: 612-618.

Colorni, A., M. Dorigo and V. Maniezzo, 1991. Distributed optimization by ant colonies. Proceedings of the European Conference on Artificial Life, (CAL' 91), Paris, France, Elsevier Publishing, pp: 134-142.

Dorigo, M. and L.M. Gambardella, 1997a. Ant colonies for the travelling salesman problem. Biosystems, 43: 73-81.

Dorigo, M. and L.M. Gambardella, 1997b. Ant colony system: A cooperative learning approach to the traveling salesman problem. IEEE Trans. Evolut. Comput., 1: 53-66. 
Dorigo, M., V. Maniezzo and A. Colorni, 1996. Ant system: Optimization by a colony of cooperative agents. IEEE Trans. Syst. Man, Cybern. B, Cybern., 26: 29-41

Laoboonlur, P., 2004. Production on scheduling in knitted fabric. Faculty of North Carolina State University.
Sheen, J.N., C.S. Chen and J.K. Yang, 1994. Time-ofuse pricing for load management programs in Taiwan Power Company. IEEE Trans. Power Syst., 9: 388-396. 